\title{
Association of lung and heart dose with survival in patients with non-small cell lung cancer underwent volumetric modulated arc therapy
}

This article was published in the following Dove Press journal: Cancer Management and Research

\author{
Lanxiao Shen ${ }^{1, *}$ \\ Cong $\mathrm{Liu}^{2} *$ \\ Juebin Jin $^{3}$ \\ Ce Han' \\ Yongqiang Zhou' \\ Xiaomin Zheng' \\ Changfei Gong' \\ Mengfeng Chen ${ }^{4}$ \\ Congying $\mathrm{Xie}^{1}$ \\ Xiance Jin ${ }^{\prime}$
}

'Radiation and Medical Oncology

Department, The First Affiliated Hospital of Wenzhou Medical University,

Wenzhou 325000, People's Republic of

China; ${ }^{2}$ Ningbo Institute of Technology,

Zhejiang University, Ningbo 315100,

People's Republic of China; ${ }^{3}$ Department of Medical Engineering, The Ist Affiliated Hospital of Wenzhou Medical University, Wenzhou 325000, People's Republic of

China; ${ }^{4}$ Respiratory Department, Third People's Hospital of Yueqing, Wenzhou

325600, People's Republic of China

*These authors contributed equally to this work

\begin{abstract}
Background: Controversial conclusions had been reported in studies trying to confirm the impact of heart dose on overall survival (OS) reported in RTOG 0167 for non-small cell lung cancer (NSCLC) patients who underwent radiotherapy (RT). The purpose of this study is to investigate the association of lung and heart dosimetric parameters with OS in NSCLC patients treated by volumetric modulated arc therapy (VMAT).

Methods: Inoperable NSCLC patients treated by VMAT from March 2012 to December 2015 were retrospectively reviewed. OS and progression-free survival (PFS) were estimated with the Kaplan-Meier method. Univariate and multivariate analyses were conducted with Cox proportional hazards model. Multivariate model building was conducted using stepwise regression for variables with $p$-value smaller than 0.2 in the univariate analysis.

Results: There were 130 NSCLC patients enrolled in this study with a median age of 63 years (range from 34 to $82 \mathrm{y}$ ). The median prescription dose for these patients was $56 \mathrm{~Gy}$ (range 40-70 Gy) with a mean heart and lung dose of $14.8 \pm 8.5 \mathrm{~Gy}$ and $13.6 \pm 4.4 \mathrm{~Gy}$, respectively. The rates of patients with above grade III radiation pneumonitis (RP) and fibrosis were $8.5 \%$ and $8.5 \%$, respectively. The 2-year PFS and OS of these patients were $15.2 \%$ and $39.8 \%$, with a median PFS and OS of 7.2 and 18.8 months, respectively. RP was correlated with OS $(p=0.048)$ and lung V20 was associated with PFS $(p=0.04)$ according to the univariate analysis. Multivariate analysis demonstrated that RP (HR 1.39, 95\%CI 1.010-1.909, $p=0.043$ ) and heart V15 (HR 1.02, 95\%CI $1.006-1.025 p=0.002$ ) were progression factors of OS, and no factor was associated with PFS. Conclusions: RP and heart V15 were associated with OS for patients with stage III NSCLC who underwent VMAT. Heart and lung dosimetric parameters were highly correlated with each other, sparing of heart and lung should be considered equally during the treatment planning. Keywords: non-small cell lung cancer, volumetric modulated arc therapy, heart dosimetry, radiation pneumonitis, overall survival
\end{abstract}

\section{Introduction}

The fact that high radiation dose to thoracic cancer can lead to heart toxicity has long been recognized with reported up to $20 \%$ of the patients with breast and lymphoma cancer elevated rates of heart disease typically 1 or more years after radiotherapy (RT). ${ }^{1-3}$ Due to few long-term survivors of patients with advanced non-small cell lung cancer (NSCLC), the clinical relevance of RT-associated heart disease for these patients is unclear. Recently, the landmark trial (radiation therapy oncology group (RTOG) 0617), which compared high-dose versus standard-dose thoracic radiation for patients with stage IIIA-IIIB NSCLC and closed early due to
Correspondence: Congying Xie; Xiance Jin Radiation and Medical Oncology

Department, The Ist Affiliated Hospital of Wenzhou Medical University, Wenzhou 325000, People's Republic of China Tel +865778806 9316;

+8657788069370

Fax +86 5775557899961 I88I;

+8657755578999664166

Email wzxiecongying@163.com; jinxc1979@hotmail.com 
futility boundaries were crossed, reported that heart volume receiving 5 Gy (V5) or 30 Gy (V30) was a significant negative effect on survival. ${ }^{4}$ However, the heart dose differences between two arms and specific cardiac toxicities were not reported.

Lots of studies were conducted to investigate whether the impact of heart dose on early overall survival (OS) reported in RTOG0617 could be confirmed in an independent cohort. $^{5-9}$ Tucker et al retrospectively reviewed 465 patients with stage IIIA-IIIB NSCLC received 3D conformal radiotherapy (CRT), intensity-modulated radiotherapy (IMRT), or proton therapy with concurrent chemotherapy between 1999 and 2010 and reported that heart dose was not associated with early survival outcomes, but he suggested that dose to the heart should be minimized based on the known adverse effects of RT on vasculature and cardiac function..$^{5}$ On the other study, after reviewing and recontouring the normal tissues following RTOG 0617 guideline for 333 patients with locally advanced (LA) NSCLC, Speirs et al reported that heart V50 was associated with OS and cardiac toxicity for locally advanced NSCLC treated with chemoradiation. ${ }^{6}$ Wang et al reviewed six trials including 127 patients with stage III NSCLC received 70-90 Gy (median 74 Gy) RT and reported that heart doses were not associated with OS, but he suggested that cardiac toxicity after RT may occur earlier than historically understood, and heart doses should be minimized. ${ }^{7}$

These controversial conclusions indicated that the role of heart dose on the effect of OS was still unclear for patients with NSCLC who underwent RT. Studies suggested that although heart dose may be important, it may not be cardiac toxicity that is driving survival, heart dose may be a surrogate for other prognostic factors in stage III NSCLC rather than an independent predictor of outcome. ${ }^{10} \mathrm{Oh}$ et al retrospectively reviewed 453 patients with stage I-III esophageal cancer treated by definitive or preoperative chemoradiation and reported that cardiac dose was not independently predictive after adjusting for lung dose and other clinical factors of survival, but lung dose was a significant independent predictor of survival. ${ }^{11}$

Meanwhile, advanced RT modalities, such as IMRT can lower heart doses and reduce their toxicities. ${ }^{12,13}$ As an extended form of IMRT, volumetric modulated arc therapy (VMAT) is able to achieve similar dosimetric distribution while decreasing machine unit (MU) and treatment delivery time compared with IMRT; however, the clinical outcomes and related toxicities of VMAT were rarely reported. The purpose of this study is to investigate the efficacy and safety of VMAT, as well as the association of lung and heart dosimetry with survival in patients with NSCLC treated by VMAT.

\section{Materials and methods}

\section{Patients and treatment}

Patients who underwent definitive RT for stage IIIA/IIIB NSCLC with or without concurrent chemotherapy from March 2012 to November 2015 were retrospectively reviewed in this study. Concurrent chemotherapy was administered with a combination of cisplatin $50 \mathrm{mg} / \mathrm{m}^{2}$ on days $1,8,29$, etoposide $50 \mathrm{mg} / \mathrm{m}^{2}$ on days 3,30 ; or carboplatin AUC 5 on day 1 and paclitaxel $500 \mathrm{mg} / \mathrm{m}^{2}$ on day 1 , repeated every 21 days for 4 cycles. Gross tumor volume (GTV) was delineated on contrast CT images following the International Commission on Radiation Units and Measurements (ICRU) report 62 criteria. $^{14}$ Planning target volume (PTV) was considered to include the GTV plus a $10-15 \mathrm{~mm}$ margin. Normal lung volumes were contoured by excluding the GTVs. Heart was delineated according to the Atlas for organs at risk for thoracic radiation therapy. RT was delivered with VMAT technique for a prescription dose from 40 to $70 \mathrm{~Gy}$ at 2 Gy per fraction. Detailed VMAT optimization was reported in a previous paper. ${ }^{15}$ This retrospective study was approved by the Institutional Review Board (IRB) of the First Affiliated Hospital of Wenzhou Medical University. The patient data confidentiality was maintained in this study and compliance with the Declaration of Helsinki. The need for written informed consent was waived by the IRB due to the retrospective nature of this study.

\section{Dosimetric parameters}

Dosimetric parameters of PTV and organs at risk (OARs) from final treated VMAT plans were extracted from Pinnacle (Phillips, version 9.8) treatment planning system (TPS) using home built MATLAB codes. For PTV, the maximum (Dmax), mean (Dmean) dose, and the volume covered by the prescription dose (V95\%, V93\%) and homogeneity index (HI) were extracted. The Dmax and D2\% of the spinal cord, the V5, V15, V25, V30, and V50 (percent volume irradiated by X dose) of the heart, and the V5, V10, V13, V20, and V30 and Dmean of the lungs were also extracted.

\section{Follow-up}

Follow-up evaluation was conducted every 3 months for the first 2 years, and every 6 months thereafter. Patients were 
required to have an immediate examination or intervention if symptoms, such as fever, cough, or shortness of breath were observed during follow-up. Acute radiation pneumonitis (RP) and late fibrosis were diagnosed by at least two radiation oncologists with consensus according to the Common Terminology Criteria for Adverse Events (CTCAE) version 4.0. ${ }^{16} \mathrm{RP}$ was scored as greater than or equal to grade III side effects.

\section{Statistical analysis}

Progression-free survival (PFS) was calculated from the date of RT to the time of first local or distant recurrence, or death from any cause. OS was measured from the date of RT to death or the last follow-up visit. Survival curves were estimated using Kaplan-Meier method and comparisons were made using the log-rank test. Univariate and multivariate analyses were conducted with Cox proportional hazards model. Multivariate

Table I Characteristics of patients with lung cancer who underwent VMAT treatment

\begin{tabular}{|l|l|}
\hline Characteristics & All patients (N= I30) \\
\hline Median age, y (range) & $63(34-82)$ \\
\hline Sex & $100(76.9 \%)$ \\
Male & $30(23.1 \%)$ \\
\hline Female & \\
\hline Pathology & $48(36.9 \%)$ \\
Adenocarcinoma & $64(49.3 \%)$ \\
Squamous cell & $16(12.3 \%)$ \\
Mixed & $2(1.5 \%)$ \\
Others & \\
\hline Stage & $58(44.6 \%)$ \\
IIIA & $72(55.4 \%)$ \\
IIIB & \\
\hline Chemotherapy & $87(66.9 \%)$ \\
With & $43(33.1 \%)$ \\
Without & \\
\hline Radiation pnuemonitis & $16(12.3 \%)$ \\
Grade 0 & $70(53.8 \%)$ \\
Grade I & $33(25.4 \%)$ \\
Grade II & $10(7.7 \%)$ \\
Grade III & $1(0.8 \%)$ \\
Grade IV & $6(4.6 \%)$ \\
\hline Fibrosis & $69(53.1 \%)$ \\
Grade 0 & $44(33.8 \%)$ \\
Grade I & $11(8.5 \%)$ \\
Grade II & \\
Grade III & \\
\hline
\end{tabular}

Abbreviation: VMAT, volumetric modulated arc therapy. model building was conducted using stepwise regression (modification of the forward selection method) for variables with probability $p$-value smaller than 0.2 in the univariate analysis. Correlation analysis was done with Pearson's method. A $p$-value of $<0.05$ was considered statistically significant. All statistical analyses were performed using IBM SPSS version 22.0 (IBM, Armonk, NY, USA).

\section{Results}

A total of 130 patients (Male 100, female 30) with stage III NSCLC who underwent VMAT were enrolled in this study with a median age of 63 years (range from 34 to $82 \mathrm{y}$ ). Detailed characteristics of these patients are presented in Table 1. The majority of patients had adenocarcinoma (36.9\%) and squamous cell carcinoma (49.3\%) with $66.9 \%$ patients had concurrent chemotherapy. The detailed dosimetric parameters are presented in Table 2. The median prescription dose for these patients was 56 Gy (range 40-70 Gy) with a mean heart and lung dose of $14.8 \pm 8.5$ Gy and 13.6 \pm 4.4 Gy, respectively. The rates of patients with above grade III RP and fibrosis were $8.5 \%$ and $8.5 \%$, respectively. Figure 1 presents

Table 2 Dosimetric parameters of patients with lung cancer who underwent VMAT treatment

\begin{tabular}{|l|l|}
\hline Median radiation dose, Gy (range) & $\mathbf{5 6}$ (40-70) \\
\hline PTV, mean \pm SD & \\
Dmax (Gy) & $60.6 \pm 8.8$ \\
Dmean (Gy) & $55.7 \pm 0.8$ \\
V93 (\%) & $90.7 \pm 12.2$ \\
V95 (\%) & $87.4 \pm 14.0$ \\
HI & $0.02 \pm 0.01$ \\
\hline Cord, mean \pm SD & \\
Dmax (Gy) & $32.3 \pm 10.5$ \\
D2 (Gy) & $31.6 \pm 10.7$ \\
\hline Heart, mean \pm SD & \\
Dmean (Gy) & $14.8 \pm 8.5$ \\
V5 (\%) & $54.1 \pm 29.9$ \\
V15 (\%) & $37.4 \pm 24.7$ \\
V25 (\%) & $25.1 \pm 17.3$ \\
V30 (\%) & $20.1 \pm 14.0$ \\
V50 (\%) & $6.4 \pm 6.5$ \\
\hline Lung, mean $\pm S D$ & \\
Dmean (Gy) & $13.6 \pm 4.4$ \\
V5 (\%) & $59.1 \pm 19.2$ \\
V10 (\%) & $44.1 \pm 16.7$ \\
V13 (\%) & $36.4 \pm 13.7$ \\
V20 (\%) & $23.9 \pm 7.9$ \\
V30 (\%) & $15.2 \pm 5.1$ \\
\hline
\end{tabular}

Abbreviations: VMAT, volumetric modulated arc therapy; PTV, planning target volume; $\mathrm{HI}$, homogeneity index. 


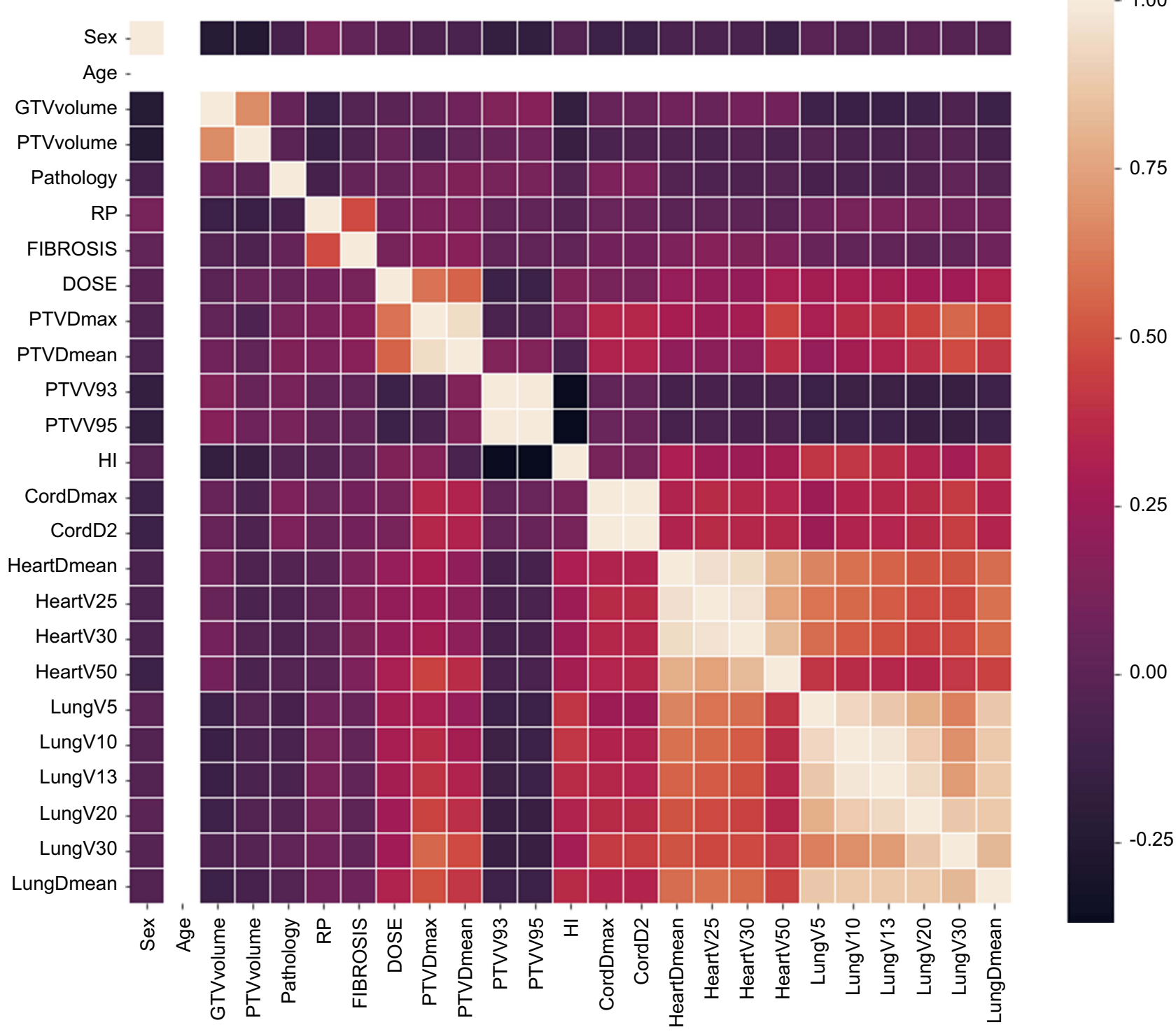

Figure I Heatmap of Pearson correlation between heart and lung dosimetric parameters.

Abbreviations: GTV, gross tumor volume; PTV, planning target volume; RP, radiation pneumonitis; HI, homogeneity index.

the Pearson correlation heatmap between all the relative parameters. Lung V5, V10, V13, and lung mean dose were correlated with heart dosimetric parameters.

PFS and OS with $95 \%$ confidence interval (CI) of these patients are shown in Figure 2 with a median follow-up of 14 months. The 2-year PFS and OS of these patients were 15.2\% and $39.8 \%$, with a median PFS and OS of 7.2 and 18.8 months, respectively. Univariate and multivariate analysis on the factors that associated with OS and PFS is presented in Table 3. RP was correlated with OS $(p=0.048)$ and lung V20 was associated with PFS $(p=0.04)$ according to the univariate analysis. Multivariate analysis demonstrated that RP (HR 1.39, 95\%CI 1.010-1.909, $p=0.043$ ) and heart V15
(HR 1.02, 95\%CI 1.006-1.025 $p=0.002$ ) were progression factors of OS, and no factors were associated with PFS. The Kaplan-Meier curve and $p$-value (0.001) from stratified Cox proportional hazards model are presented in Figure 3.

\section{Discussion}

The clinical outcome, impacts of heart and lung dosimetric parameters on the OS and PFS of patients with stage III NSCLC who underwent VMAT were investigated in this study. The RP and heart V15 were correlated with OS, while there was no heart and lung dosimetry clearly correlated with PFS. 


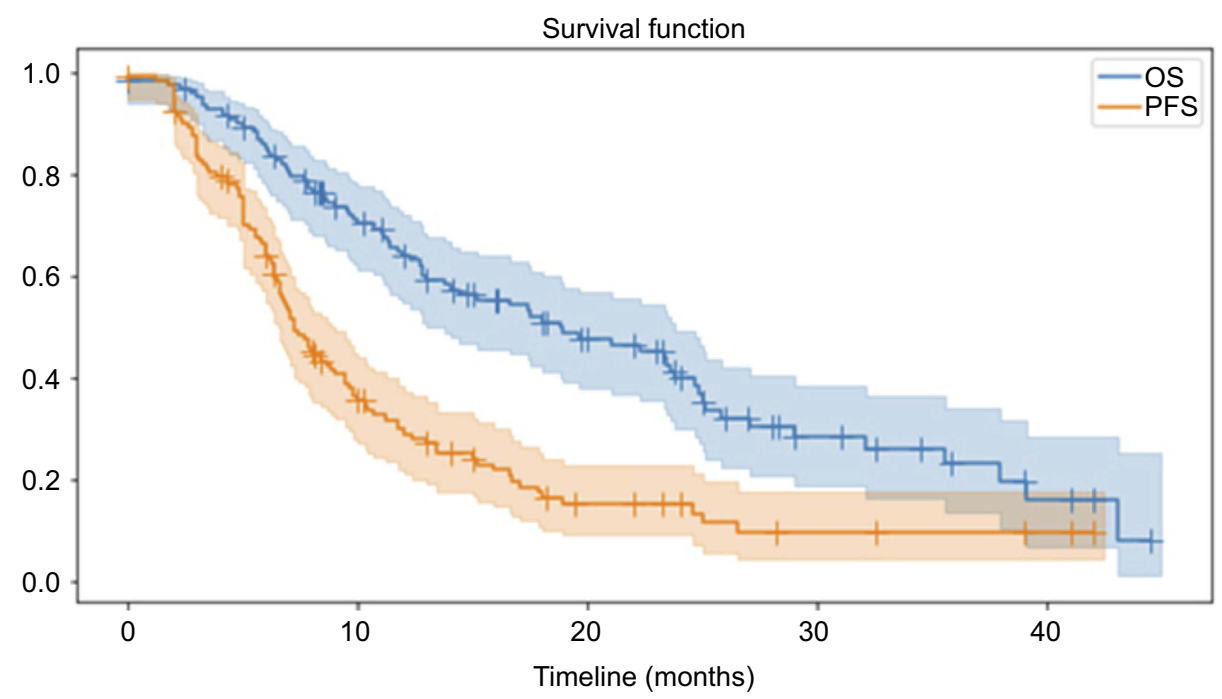

Figure 2 Progression-free survival (PFS) and overall survival (OS) with $95 \%$ confidence interval of patients with non-small cell lung cancer who underwent volumetric modulated arc therapy.

Dosimetric studies had reported that VMAT and IMRT were able to achieve superior dose distributions compared with conformal RT technique in the treatment of lung cancer and resulted in better local control due to their dose sculpting abilities. ${ }^{17}$ However, few studies had reported the clinical outcome of VMAT in the treatment of lung cancer. A 2-year PFS and OS of $15.2 \%$ and $39.8 \%$ were reported in this study for VMAT in the treatment of stage III NSCLC. Similarly, a 2-year OS of 58\% for patients with stage Ib-IIIb (62\% stage III) NSCLC and a 1 -year OS of $57 \%$ for patients with NSCLC ( $85 \%$ stage III) were reported by Sura et al and Yom et al, respectively, for patients who underwent IMRT treatment. ${ }^{18,19}$ Liao et al also reported a 2-year PFS and OS of 38\% and $46 \%$, respectively, for patients with locally advanced NSCLC treated by IMRT. ${ }^{20}$

The variations in PFS and OS for different studies mentioned earlier could be attributed to many factors, such as differences in histological type, adjuvant chemotherapy, comorbid conditions, RT techniques, etc. ${ }^{21,22}$ Treatment complications induced during RT also have a role in OS. RP is a common and lethal lung injury caused by the lung exposure during RT for patients with NSCLC. ${ }^{22}$ RP is strongly correlated with the dose irradiated to normal lung and could affect $13-37 \%$ of the patients depending on the specific RT techniques. ${ }^{23,24}$ In this study, RP (HR 1.39, 95\% CI 1.010-1.909, $p=0.043$ ) was correlated to OS in both univariate and multivariate analysis for NSCLC patients who underwent VMAT. Consistently, Inoue et al reported that severe RP was the only factor negatively associated with survival for patients who underwent definitive radiotherapy for lung cancer. ${ }^{25}$ In another study, Minami-Shimmyo et al stated that deaths after concurrent chemoradiation therapy for patients with lung cancer were associated with RP or pulmonary fibrosis. ${ }^{26}$ Oh et al also reported that lung dose is a significant independent predictor of survival for patients with esophageal cancer who underwent radiotherapy. ${ }^{11}$

In this study, multivariate analysis indicated that heart V15 (HR 1.02) was a weak progression factor of OS for NSCLC patients who underwent VMAT. This weak correlation could not further support the statements reported in the previous studies that V5, V30, or V50 were associated with OS of patients with lung cancer who underwent radiotherapy. ${ }^{4,6}$ Concerns and reports regarding radiationrelated cardiac and vascular toxicity were mainly raised in the era when two-dimensional RT was applied to treat the patients with breast cancer and Hodgkin's lymphoma and severe complications were observed. ${ }^{27,28}$ Currently, with the wide application of IMRT and VMAT, heart exposure andthe risk of radiation-related events had been steadily declined.${ }^{29,30}$ On the other hand, heart and lung dosimetric parameters tend to be highly correlated with each other, as demonstrated by the Pearson correlation results in Figure 1 in this study. Similarly, Tucker et al explained that the effect of heart dose on OS might be due to a correlation between heart and lung doses in their study and in the study of RTOG $0167 .{ }^{5}$

Although we found that heart dose was weakly correlated with survival in this study, sparing of the heart should remain a priority during clinical practice in the RT of lung cancer, as 
Table 3 Univariate and multivariate analysis of factors associated with overall survival and progression-free survival with Cox model

\begin{tabular}{|c|c|c|c|c|c|c|c|c|c|}
\hline \multirow{3}{*}{ Characteristics } & \multicolumn{6}{|l|}{ os } & \multirow{2}{*}{\multicolumn{3}{|c|}{$\begin{array}{l}\text { PFS } \\
\text { Univariate analysis }\end{array}$}} \\
\hline & \multicolumn{3}{|c|}{ Univariate analysis } & \multicolumn{3}{|c|}{ Multivariate analysis } & & & \\
\hline & HR & $95 \% \mathrm{Cl}$ & $p$ & HR & $95 \% \mathrm{Cl}$ & $p$ & HR & $95 \% \mathrm{Cl}$ & $p$ \\
\hline Age & 1.02 & $0.987-1.043$ & 0.29 & & & & 0.99 & $0.966-1.013$ & 0.36 \\
\hline Sex & 1.53 & $0.780-2.99 \mid$ & 0.22 & & & & 1.25 & $0.688-2.253$ & 0.47 \\
\hline Pathology & 1.19 & $0.87 I-1.62 I$ & 0.28 & & & & 0.92 & $0.705-1.199$ & 0.36 \\
\hline Chemotherapy & 1.26 & $0.658-2.415$ & 0.49 & & & & 1.19 & $0.690-2.064$ & 0.53 \\
\hline Radiation pnuemonitis & 1.54 & $1.004-2.364$ & 0.048 & 1.39 & $1.010-1.909$ & 0.043 & 1.14 & $0.794-1.639$ & 0.48 \\
\hline Fibrosis & 1.12 & $0.728-1.734$ & 0.60 & & & & 1.25 & $0.840-1.857$ & 0.27 \\
\hline Prescription dose & 1.00 & $0.999-1.001$ & 0.93 & & & & 1.00 & $0.999-1.003$ & 0.65 \\
\hline \multicolumn{10}{|l|}{ PTV } \\
\hline Dmax & 1.00 & $0.998-1.003$ & 0.99 & & & & 1.00 & $0.999-1.003$ & 0.25 \\
\hline Dmean & 1.00 & $0.997-1.002$ & 0.81 & & & & 0.99 & $0.997-1.001$ & 0.19 \\
\hline V93 & 0.91 & $0.747-1.110$ & 0.35 & & & & 0.97 & $0.84 I-1.109$ & 0.62 \\
\hline V95 & 1.12 & $0.952-1.308$ & 0.18 & & & & 1.06 & 0.948-1.191 & 0.30 \\
\hline \multicolumn{10}{|l|}{ Cord } \\
\hline Dmax & 1.00 & $0.993-1.007$ & 0.99 & & & & 1.00 & $0.995-1.008$ & 0.65 \\
\hline D2 & 1.00 & $0.993-1.007$ & 0.95 & & & & 1.00 & $0.992-1.005$ & 0.60 \\
\hline \multicolumn{10}{|l|}{ Heart } \\
\hline Dmean & 1.00 & $0.998-1.002$ & 0.97 & & & & 1.00 & $0.999-1.003$ & 0.32 \\
\hline V5 & 0.99 & $0.959-1.022$ & 0.52 & & & & 0.98 & $0.956-1.013$ & 0.28 \\
\hline V15 & 1.04 & $0.985-1.093$ & 0.17 & 1.02 & $1.006-1.025$ & 0.002 & 1.03 & $0.980-1.082$ & 0.25 \\
\hline V25 & 1.03 & $0.934-1.135$ & 0.56 & & & & 0.91 & $0.745-1.106$ & 0.28 \\
\hline V30 & 0.93 & $0.845-1.012$ & 0.089 & & & & 1.07 & $0.907-1.262$ & 0.42 \\
\hline V50 & 1.09 & $0.989-1.194$ & 0.083 & & & & 0.95 & $0.895-1.084$ & 0.76 \\
\hline \multicolumn{10}{|l|}{ Lung } \\
\hline V5 & 1.03 & $0.960-1.094$ & 0.47 & & & & 0.99 & $0.923-1.054$ & 0.68 \\
\hline VIO & 0.86 & $0.706-1.035$ & 0.11 & & & & 0.99 & $0.775-1.167$ & 0.63 \\
\hline $\mathrm{V} 13$ & 1.12 & $0.879-1.429$ & 0.36 & & & & 0.95 & $0.753-1.236$ & 0.78 \\
\hline V20 & 1.04 & $0.845-1.288$ & 0.69 & & & & 1.22 & $1.013-1.470$ & 0.04 \\
\hline V30 & 0.98 & $0.829-1.167$ & 0.85 & & & & 0.89 & $0.757-1.050$ & 0.17 \\
\hline Dmean & 1.00 & $0.999-1.003$ & 0.23 & & & & 1.00 & $0.998-1.004$ & 0.41 \\
\hline
\end{tabular}

Abbreviations: PTV, planning target volume; OS, overall survival; PFS, progressive-free survival.

many evidence indicated that cardiac exposure was associated with long-term cardiac disease and contribute to morbidity and even mortality. ${ }^{31}$ One limitation of this study is that this is a retrospective study from one institution only; the impact of different modern RT techniques other than VMAT and dosimetric constraints of other OARs on survival needs further investigation.

\section{Conclusions}

In summary, RP and heart V15 were associated with OS for patients with stage III NSCLC who underwent VMAT. Heart and lung dosimetric parameters were highly correlated with each other, sparing of heart and lung should be considered equally during the treatment planning. 


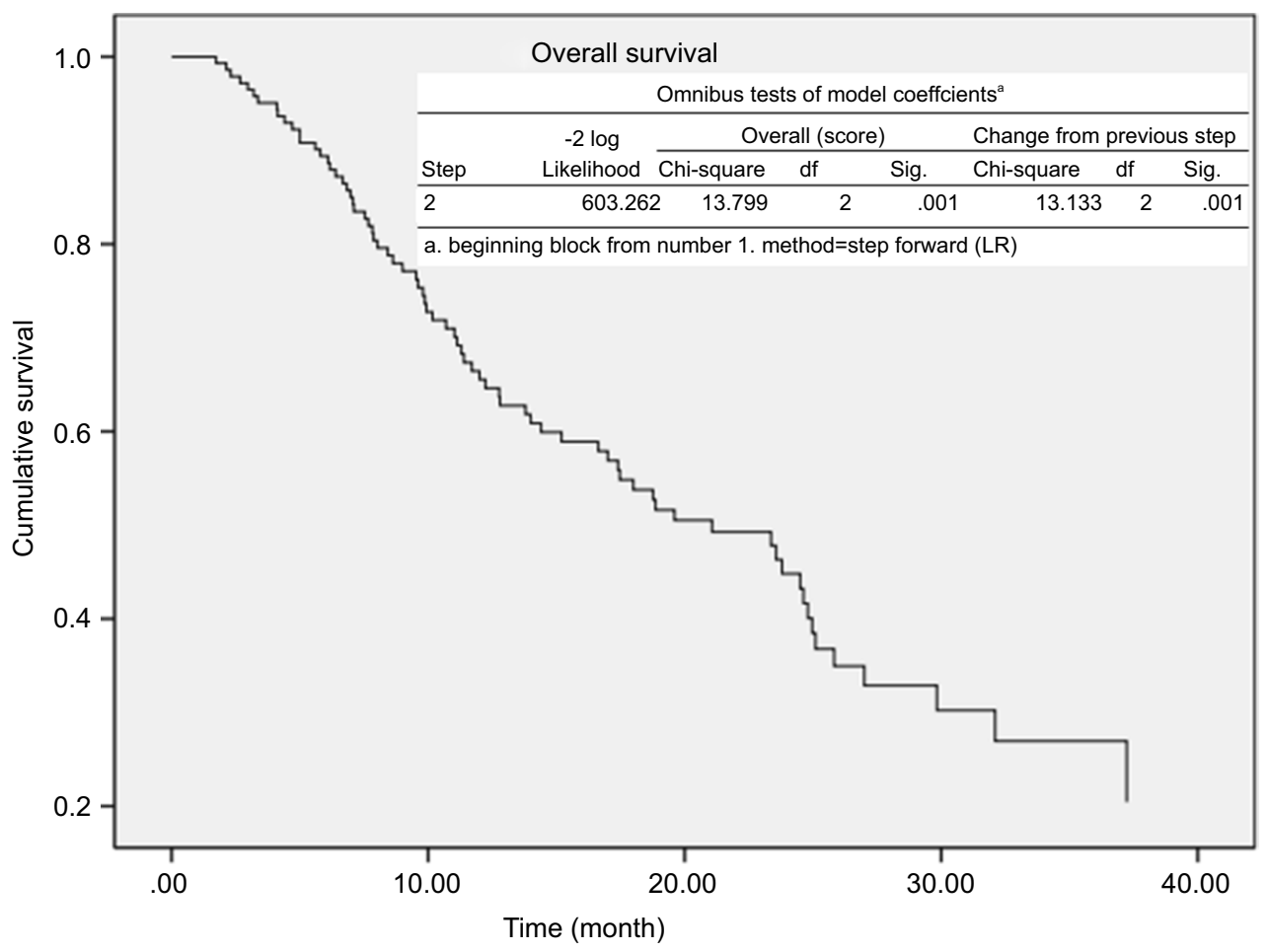

Figure 3 The Kaplan-Meier curve and p-value (0.00I) from stratified Cox proportional hazards model.

\section{Acknowledgments}

This work was supported by funding of National Natural Science Foundation of China (11675122) and Wenzhou Municipal Science and Technology Bureau (2018ZY016).

\section{Disclosure}

The authors report no conflicts of interest in this work.

\section{References}

1. Hooning MJ, Botma A, Aleman BM, et al. Long-term risk of cardiovascular disease in 10-year survivors of breast cancer. J Natl Cancer Inst. 2007;99:365-375. doi:10.1093/jnci/djk064

2. van Nimwegen FA, Schaapveld M, Janus CP, et al. Cardiovascular disease after Hodgkin lymphoma treatment: 40-year disease risk. JAMA Intern Med. 2015;175:1007-1017. doi:10.1001/jamainternmed.2015.1180

3. van Nimwegen FA, Schaapveld M, Cutter DJ, et al. Radiation doseresponse relationship for risk of coronary heart disease in survivors of Hodgkin lymphoma. J Clin Oncol. 2016;34:235-243. doi:10.1200/ JCO.2015.63.4444

4. Bradley JD, Paulus R, Komaki R, et al. Standard-dose versus highdose conformal radiotherapy with concurrent and consolidation carboplatin plus paclitaxel with or without cetuximab for patients with stage IIIA or IIIB non-small-cell lung cancer (RTOG 0617): a randomised, two-by-two factorial phase 3 study. Lancet Oncol. 2015;16:187-199.

5. Tucker SL, Liu A, Gomez D, et al. Impact of heart and lung dose on early survival in patients with non-small cell lung cancer treated with chemoradiation. Radiother Oncol. 2016;119(3):495-500. doi:10.1016/j. radonc.2016.04.025
6. Speirs CK, DeWees TA, Rehman S, et al. Hart dose is an independent dosimetric predictor of OS in LA NSCLC. J Thorac Oncol. 2017;12 (2):293-301. doi:10.1016/j.jtho.2016.09.134

7. Wang K, Eblan MJ, Deal AM, et al. Marks cardiac toxicity after radiotherapy for stage III non-small-cell lung cancer: pooled analysis of dose-escalation trials delivering 70 to 90 Gy. J Clin Oncol. 2017;35(13):1387-1394. doi:10.1200/JCO.2016.70.0229

8. Stam B, van der Bijl E, van Diessen J, et al. Heart dose associated with overall survival in locally advanced NSCLC patients treated with hypofractionated chemoradiotherapy. Radiother Oncol. 2017;125(1):62-65. doi:10.1016/j.radonc.2017.09.004

9. McWilliam A, Kennedy J, Hodgson C, et al. Radiation dose to heart base linked with poorer survival in lung cancer patients. Eur $J$ Cancer. 2017;85:106-113. doi:10.1016/j.ejca.2017.07.053

10. Cella L, D'Avino V, Palma G, et al. Modeling the risk of radiation-induced lung fibrosis: irradiated heart tissue is as important as irradiated lung. Radiother Oncol. 2015;117(1):36-43. doi:10.1016/j.radonc.2015.07.051

11. Oh P, Zhang M, Brady P, et al. Impact of lung and heart dose on survival after radiotherapy for esophageal cancer. J Clin Oncol. 2018;36(4 suppl):3. doi:10.1200/JCO.2018.36.4_suppl.3

12. Sejpal S, Komaki R, Tsao A, et al. Early findings on toxicity of proton beam therapy with concurrent chemotherapy for nonsmall cell lung cancer. Cancer. 2011;117(13):3004-3013. doi:10.1002/cncr.25848

13. Chang JY, Zhang X, Wang X, et al. Significant reduction of normal tissue dose by proton radiotherapy compared with three-dimensional conformal or intensity-modulated radiation therapy in Stage I or Stage III non-small-cell lung cancer. Int J Radiat Oncol Biol Phys. 2006;65(4):1087-1096. doi:10.1016/j.ijrobp.2006.01.052

14. International Commission on Radiation Units and Measurements. ICRU Reports 62 (Supplement to ICRU Report 50). Prescribing, Recording, and Reporting Photon Beam Therapy. Bethesda (MD): ICRU; 1999.

15. Jin X, Xie C. Dosimetric and clinical benefits of conformal radiotherapy plus volumetric modulated arc therapy in the treatment of non-small cell lung cancer. Med Phys. 2015;42(6):3704-3705. doi: $10.1118 / 1.4926135$ 
16. Chen AP, Setser A, Anadkat MJ, et al. Grading dermatologic adverse events of cancer treatments: the common terminology criteria for adverse events version 4.0. J Am Acad Dermatol. 2012;6(7):10251039. doi:10.1016/j.jaad.2012.02.010

17. Bree I, van Hinsberg MG, van Veelen LR. High-dose radiotherapy in inoperable non-small cell lung cancer: comparison of volumetric modulated arc therapy, dynamic IMRT and 3D conformal radiotherapy. Med Dosim. 2012;37:353-357. doi:10.1016/j.meddos.2011.12.002

18. Sura S, Gupta V, Yorke E, Jackson A, Amols H, Rosenzweig KE. Intensity-modulated radiation therapy (IMRT) for inoperable nonsmall cell lung cancer: the Memorial Sloan-Kettering Cancer Center (MSKCC) experience. Radiother Oncol. 2008;87:17-23. doi:10.1016/j.radonc.2008.02.005

19. Yom SS, Liao Z, Liu HH, et al. Initial evaluation of treatment-related pneumonitis in advanced-stage non-small-cell lung cancer patients treated with concurrent chemotherapy and intensity-modulated radiotherapy. Int $J$ Radiat Oncol Biol Phys. 2007;68:94-102. doi:10.1016/j.ijrobp.2006.1 2.031

20. Liao ZX, Komaki RR, Thames HD Jr, et al. Influence of technologic advances on outcomes in patients with unresectable, locally advanced non-small-cell lung cancer receiving concomitant chemoradiotherapy. Int J Radiat Oncol Biol Phys. 2010;76:775-781. doi:10.1016/j. ijrobp.2009.02.032

21. Nalbantov G, Kietselaer B, Vandecasteele K, et al. Cardiac comorbidity is an independent risk factor for radiation-induced lung toxicity in lung cancer patients. Radiother Oncol. 2013;109:100-106. doi:10.1016/j.radonc.2013.08.035

22. Goldstraw P, Ball D, Jett JR, et al. Non-small- cell lung cancer. Lancet. 2011;378:1727-1740. doi:10.1016/S0140-6736(11)60984-7

23. Wang S, Liao Z, Wei X, et al. Analysis of clinical and dosimetric factors associated with treatment related pneumonitis (TRP) in patients with non-small-cell lung cancer (NSCLC) treated with concurrent chemotherapy and three dimensional conformal radiotherapy (3-DCRT). Int J Radiat Oncol Biol Phys. 2006;66:13991407. doi:10.1016/j.ijrobp.2006.07.1337
24. Rancati T, Ceresoli GL, Gagliardi G, Schipani S, Cattaneo GM. Factors predicting radiation pneumonitis in lung cancer patients: a retrospective study. Radiother Oncol. 2003;67:275-283.

25. Inoue A, Kunitoh H, Sekine I, et al. Radiation pneumonitis in lung cancer patients: a retrospective study of risk factors and the long-term prognosis. Int J Radiat Oncol Biol Phys. 2001;49:649-655.

26. Minami-Shimmyo Y, Ohe Y, Yamamoto S, et al. Risk factors for treatment-related death associated with chemotherapy and thoracic radiotherapy for lung cancer. J Thorac Oncol. 2012;7:177-182. doi:10.1097/JTO.0b013e31823c4c07

27. Cosset JM, Henry-Amar M, Girinski T, Malaise E, Dupouy N, Dutreix J. Late toxicity of radiotherapy in Hodgkin's disease. The role of fraction size. Acta Oncol. 1988;26:123-129. doi:10.3109/ 02841868809090332

28. Brenner DJ, Shuryah I, Jozsef G, DeWyngaert KJ, Formenti SC. Risk and risk reduction of major coronary events associated with contemporary breast RT. JAMA Intern Med. 2014;174:158-160. doi:10.1001/jamainternmed.2013.11790

29. Maraldo MV, Brodin P, Aznar MC, et al. Doses to carotid arteries after modern radiation therapy for Hodgkin lymphoma: is stroke still a late effect of treatment? Int J Radiat Oncol Biol Phys. 2013;87:297-303. doi:10.1016/j.ijrobp.2013.06.004

30. Maraldo MV, Brodin NP, Aznar MC, et al. Estimated risk of cardiovascular disease and secondary cancers with modern highly conformal radiotherapy for early-stage mediastinal Hodgkin lymphoma. Ann Oncol. 2013;24:2113-2118. doi:10.1093/annonc/ mdt 156

31. Hatakenaka M, Yonezawa M, Nonoshita T, et al. Acute cardiac impairment associated with concurrent chemoradiotherapy for esophageal cancer: magnetic resonance evaluation. Int J Radiat Oncol Biol Phys. 2012;83:e67-e73. doi:10.1016/j.ijrobp.2011.12.018

\section{Publish your work in this journal}

Cancer Management and Research is an international, peer-reviewed open access journal focusing on cancer research and the optimal use of preventative and integrated treatment interventions to achieve improved outcomes, enhanced survival and quality of life for the cancer patient.
The manuscript management system is completely online and includes a very quick and fair peer-review system, which is all easy to use. Visit http://www.dovepress.com/testimonials.php to read real quotes from published authors 